\title{
A real x-y Microbulk Micromegas with segmented mesh
}

\author{
Theodoros Geralis ${ }^{1}$ \\ NCSR Demokritos, Ag. Paraskevi, Athens, Greece \\ E-mail: geral@inp.demokritos.gr
}

Martyn Davenport, Rui De Oliveira, Serge Ferry

CERN, PPE, Geneva, Switzerland

\author{
Athanasios Kalamaris, Chrysoula Vassou \\ NCSR Demokritos, Ag. Paraskevi, Athens, Greece
}

Esther Ferrer Ribas, Mariam Kebbiri, Thomas Papaevangelou

IRFU Saclay, Gif sur Yvette, France

Francisco Aznar ${ }^{2}$, Theopisti Dafni, Francisco J. Iguaz

Laboratorio De Física Nuclear y Astropartículas, University of Zaragoza, Cerbuna, Zaragoza, Spain

We present for the first time, the development of Micromegas detectors based on Microbulk technology with segmented mesh. The space charge produced within the amplification volume induces two signals: the mesh strips provide the y coordinate while the anode strips the $\mathrm{x}$ coordinate. The manufacturing of a segmented mesh simplifies the $x-y$ readout that up to now was produced in a complicated and delicate way due to the $x-y$ strips formation and had a high risk of deteriorating the detector quality or even damaging the detector in the last stages of construction. The design and manufacturing has been optimized and produced segmented mesh Microbulk Micromegas with excellent properties in energy resolution and stability. We have designed appropriate FE-electronics for providing the bias HV to every individual mesh strip and reading it out. The design aims to an ultra low background, ultra low threshold detector appropriate for rare event searches, thanks to its low material budget that may further improve the excellent Microbulk technology background properties close to $\sim$ (few) $\times 10^{-7} \mathrm{cnts} / \mathrm{keV} / \mathrm{cm}^{2} / \mathrm{s}$. We will present details of the design and the manufacturing of the segmented mesh microbulk, results on the detector performance, prospects for further improvements and possible applications in rare event searches, neutron detection and other applications. We believe that this design constitutes a break-through in the Micro Pattern Gaseous Detectors developments.

Technology and Instrumentation in Particle Physics 2014

2-6 June, 2014

Amsterdam, the Netherlands

\footnotetext{
${ }^{1}$ Speaker

2 present adress: Centro Universitario de la Defensa. Universidad de Zaragoza, Ctra. de Huesca s/n, 50090 Zaragoza, Spain.
} 


\section{Introduction}

Microbulk micromegas [1] is already a well established technology that has produced detectors with excellent characteristics in energy resolution, stability and extremely low background levels. Reference [1] presents all the details for manufacturing microbulk micromegas detectors and their main properties. Microbulk micromegas have been proven very adequate for rare event searches [2] thanks to their very low background rates, close to about $\sim 10^{-6} \mathrm{cnts} / \mathrm{keV} / \mathrm{cm}^{2} / \mathrm{s}$ [3], and their excellent energy resolution at the $\mathrm{keV}$ range. The detection and the track determination of particles suffering total absorption in the chamber sets the requirement to provide 3-dimensional event reconstruction which implies a two dimensional readout operating in TPC mode. The first designs of $\mathrm{x}$ and $\mathrm{y}$ readout layout were based on a complicated topology that was delicate and fragile during manufacturing. The anode plane was in the form of square pads which form the $\mathrm{x}$ and the $\mathrm{y}$ strips using conductive vias to two different planes behind the anode plane. A microbulk was built performing a sequence of operations: construction of readout strips on a $50 \mu \mathrm{m} \mathrm{Cu}$ double coated kapton foil using photolithography, construction of readout lines, etching of kapton and vias construction and finally photochemical production of mesh holes, kapton etching and cleaning. Interspacing of kapton foils is used wherever needed. The construction of the $\mathrm{x}$ and $\mathrm{y}$ strips is adding extra material near the sensitive volume. The whole process is delicate and complicated due to the $x-y$ strips formation and there is a high risk of deteriorating the detector quality or even damaging the detector in the last stages of construction is implicated. The manufacturing takes one man month of work.

Our research project aimed at building Microbulk detectors with segmented mesh in order to provide a simpler $\mathrm{x}$ and $\mathrm{y}$ readout and at the same time have a better determination of those coordinates as originating from the same space charge inside the amplification area. The challenge was to achieve this task while maintaining (if not improving) the good Microbulk properties. The real x-y segmented mesh microbulk micromegas further reduces the material near the sensitive detector area, because of the elimination of the $x-y$ structure with the metalized vias and the kapton layers and consists only of two $5 \mu \mathrm{m} \mathrm{Cu}$ foils and the supporting kapton (as spacer material) in between. We have performed optimization studies of the geometry in order to obtain the electric fields and the voltage settings in the conversion and the amplification volumes that would lead to optimal electron transparencies. The mesh segmentation required designing and building special front-end electronics in order to provide the HV bias individually and readout the y-strips signals. The lack of a common trigger signal further requires auto-triggering electronics to read out the strips. We have performed studies on the gain, the mesh transparency, the operation of the strips and have determined the optimum mesh and drift electric field settings. We have achieved excellent energy resolution and have identified the cause of slightly different behavior in different detectors. 


\section{Design study optimization and manufacturing}

For the proof of principle phase we decided to build small size detectors that would facilitate both the manufacturing and the tests of the first segmented mesh micromegas. Based on the past experience of the microbulk micromegas operation the holes of the mesh were limited only on top of the strips, thus obtaining better stability and charge collection. Figure 1 shows the mesh grid layout. The mesh holes diameter is $40 \mu \mathrm{m}$, the mesh (amplification) gap width is $50 \mu \mathrm{m}$ and the initial strip gap is set to $100 \mu \mathrm{m}$.

The manufacturing of the segmented microbulk is based on a double side copper clad 50 $\mu \mathrm{m}$ polyimide foil. The creation of the grid holes follows: photoresistive liquid spinning, masking, exposure, development in clean room and finally chemical etching. The creation of the cavities of the amplification volume is done by etching with sprayer. The produced amplification volumes are in the form of cylinders under the mesh holes with slightly larger diameter than the holes. In the next stage the $\mathrm{x}$ and the $\mathrm{y}$ strips formation takes place. This includes photoresistive lamination, masking exposure development and finally chemical etching. The $\mathrm{x}$ and $\mathrm{y}$ strips are identical, apart from the fact that the mesh holes are developed on the y strips. The last stage includes cleaning and passivation and electrical tests at $600 \mathrm{~V}$.
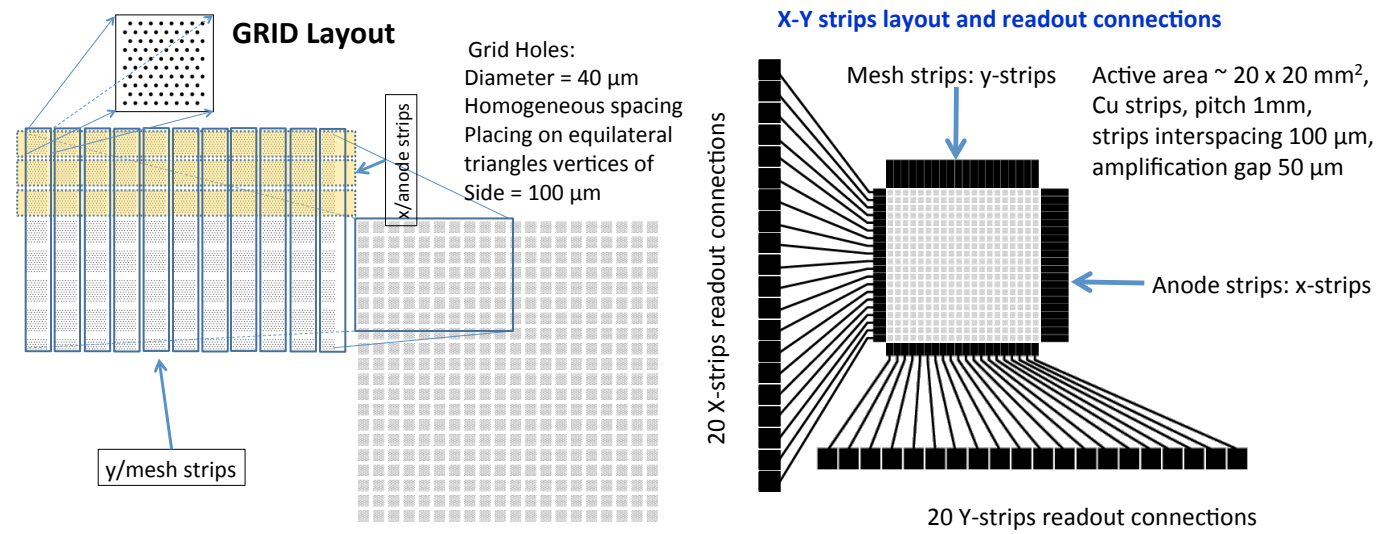

Figure 1. Grid (left) and strips layout (right) of the real x-y segmented mesh microbulk
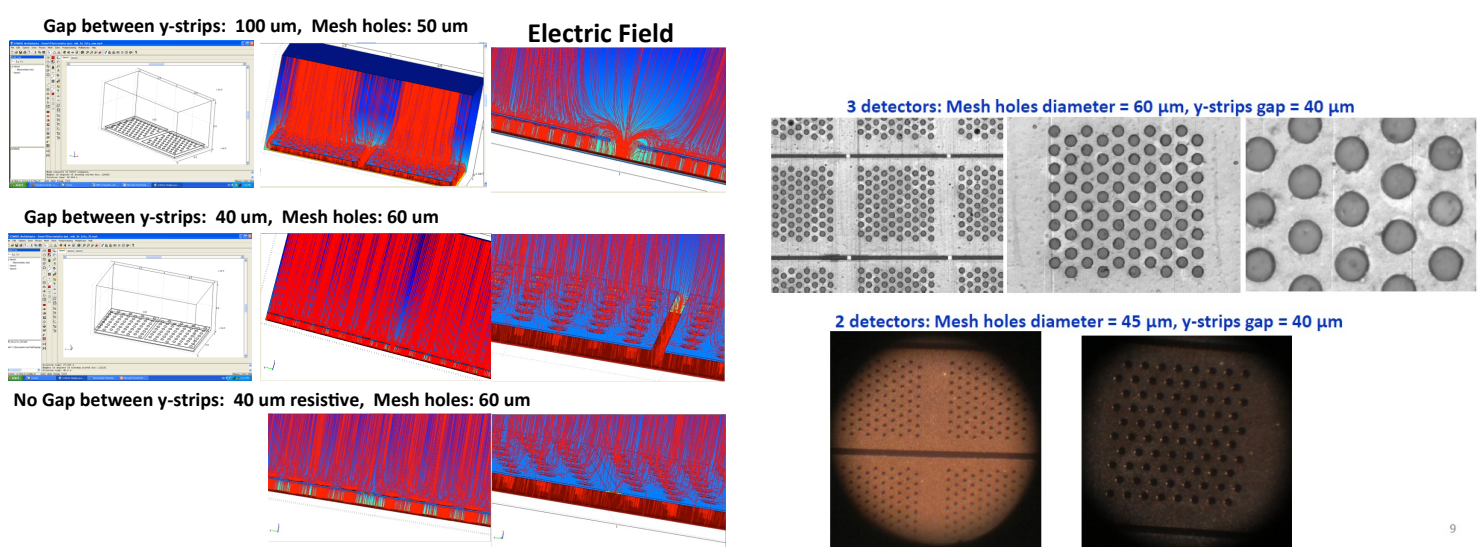

Figure 2. Left: Electric field lines at the conversion and the amplification area: Mesh gap at 100 $\mu \mathrm{m}$ (upper row images), mesh gap at $40 \mu \mathrm{m}$ (middle row images) and covered with resistive (lower row images). Right: Photos of the most recent segmented microbulk production series. 
The Comsol package [4] was used to optimize the detector geometrical characteristics in order to obtain a homogeneous electric field in both the conversion and the amplification area. Figure 2a shows a comparison between three layouts: two with different mesh interstrips spacing $(100 \mu \mathrm{m}$ and $40 \mu \mathrm{m})$ and one with resistive material between the mesh strips. Reducing the mesh strips gap to $40 \mu \mathrm{m}$ largely improves the field shape, which is perfect if we use the resistive coating. We have proceeded with the reduction of the gap to $40 \mu \mathrm{m}$, while a further reduction to $30 \mu \mathrm{m}$ will be tried in the future even though there is higher risk to produce short circuits between the strips. Figure $2 \mathrm{~b}$ shows photos of the mesh of the most recent segmented Microbulk production series. Three detectors were built with mesh holes diameter of $60 \mu \mathrm{m}$ and two of $45 \mu \mathrm{m}$. The strips gap was at $40 \mu \mathrm{m}$ for all the detectors. Improvements in the manufacturing process lead to well functioning detectors in the $3^{\text {rd }}$ production series.

\section{The mesh y-strips FE electronics}

The mesh y-strips need to be supplied by the bias HV and be read out at the same time to provide the y information. A special FE readout card was designed (see Figure 3) and four cards have been already built. The circuit has the following characteristics (see Figure 3): 1) Readout up to 64 strips, 2) HV supply for every strip independently, 3) Selection of HV supplied strips individually, 4) Transient voltage suppression (TVS) protection and 5) Single-channel read-out and all-channel read-out.

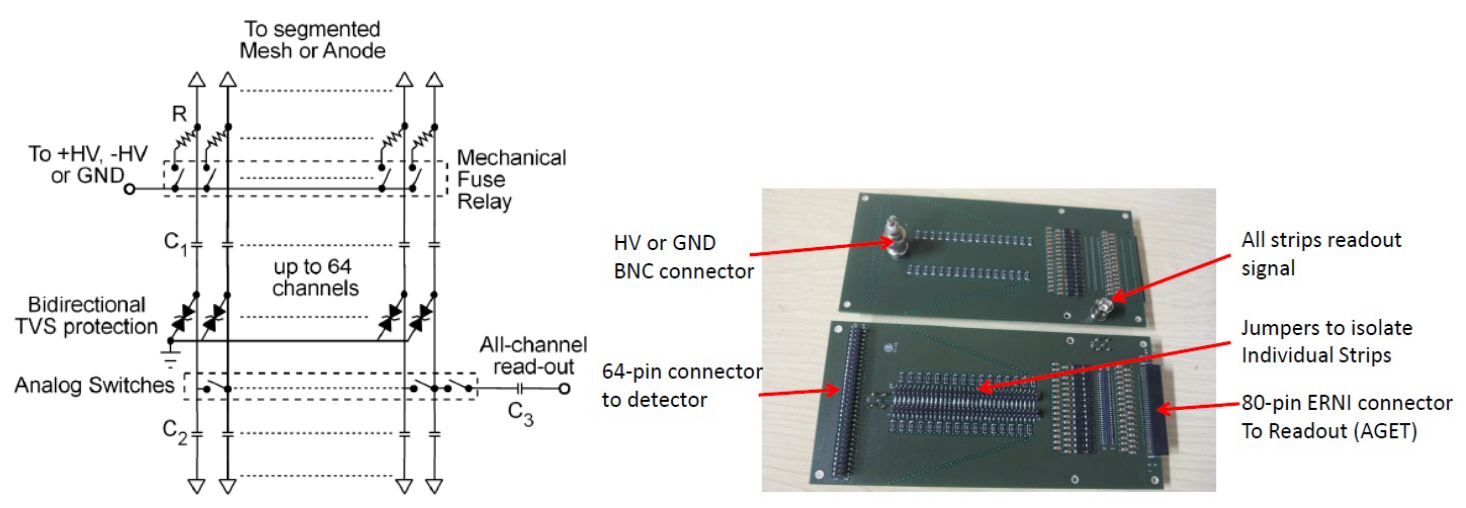

Figure 3. Schematic of the mesh y-strips readout (left) and the front and back face of the HV card (right).

Figure 3 (right) shows the front and the back side of a HV FE card. The signals are read out from the detector via the 64-pin connector while the 80-pin ERNI connector sends the strip signals to the AGET readout [5]. The cards can read out both types of strips (x anode strips and the y-strips on HV). There is also the possibility to mask individual channels. Due to the non availability of the AGET electronics at the time of the first tests, we have not performed the full detector readout yet, but only separate readout of either the $\mathrm{x}$-strips or the $\mathrm{y}$-strips at a time. 


\section{Segmented mesh microbulk characterization}

Special housing and pcb were designed and built in order to characterize the segmented Microbulk and further perform studies by reading out the strips. Figure 4 shows the raw detector, the pcb design, the placing of the segmented Microbulk in the cage together with the connection to the readout connector and the drift electrode.
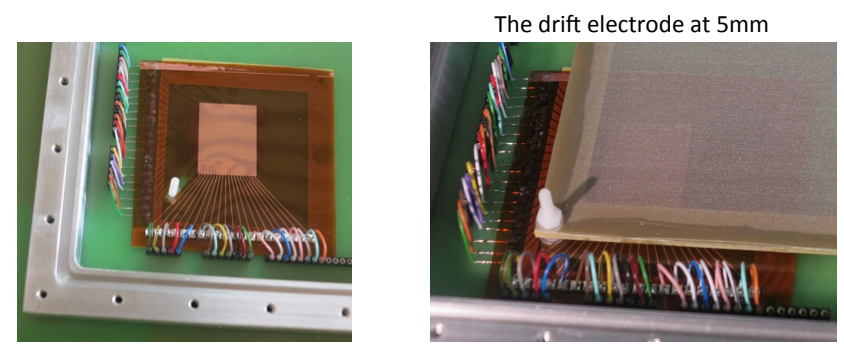

Figure 4. The raw detector and the detector housing with the drift electrode

Full characterization of the detectors took place, which consisted in studying: 1) the functionality of both $\mathrm{x}$ and $\mathrm{y}$ strips, 2) the gain, the transparency curves and the energy resolution, 3) the $\mathrm{X}$ and $\mathrm{Y}$ strips readout with the Gassiplex FE card.

\section{1 $\mathrm{X}$ and $\mathrm{Y}$ strips functionality}

We have tested the charge collection of the $\mathrm{x}$-strips and the $\mathrm{y}$-strips separately. The detector was flushed with a continuous flow of $\mathrm{Ar}+$ Isobutane $5 \%$ gas mixture during all the tests presented in this document. The y-strips common readout (short circuited) is performed by setting a "regular" HV Bias in which the HV is applied on the mesh and the anode remains at ground i.e. $\mathrm{V}_{\text {drift }}=-360 \mathrm{~V}, \mathrm{~V}_{\text {mesh }}=-330 \mathrm{~V}$ and $\mathrm{V}_{\text {anode }}=0 \mathrm{~V}$. The readout chain consists of a preamplifier (EG\&G Ortec 142IH), an amplifier (Tennelec 244) and a multichannel analyzer (Amptek MCA $8000 \mathrm{~A}$ ). Figure 5 (Left) shows the spectrum acquired using a ${ }^{55} \mathrm{Fe}$ source. The $\mathrm{x}$-strips common readout (short circuited) is performed by shifting the voltages in order to have the mesh at ground and a positive $\mathrm{HV}$ on the anode i.e $\mathrm{V}_{\text {drift }}=-30 \mathrm{~V}, \mathrm{~V}_{\text {mesh }}=0 \mathrm{~V}$ and $\mathrm{V}_{\text {anode }}=+330 \mathrm{~V}$. We call this "reversed" bias as the anode is on HV. The same readout chain, as above, was used for the readout. Figure 5 (Right) shows the equivalent spectrum from the ${ }^{55} \mathrm{Fe}$ source. We haven't understood yet why the $\mathrm{x}$-strips spectrum is rather degraded with respect to the y-strips.
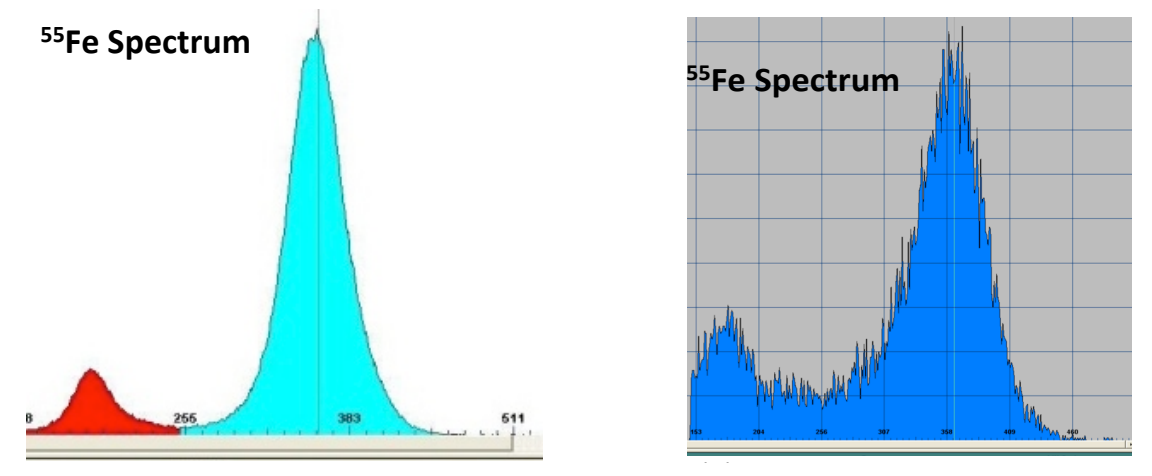

Figure 5. The ${ }^{55} \mathrm{Fe}$ spectrum for the $\mathrm{x}$ and the $\mathrm{y}$ strips of the segmented mesh detector. 


\subsection{Gain study, electron transparency and Energy resolution}

Figure 6 shows a typical gain curve for the detector version of $45 \mu \mathrm{m}$ mesh holes. The gain curves for the $60 \mu \mathrm{m}$ mesh holes version is somewhat higher due to a better optical transparency. The gain can reach operating values up to about $(5-10) \times 10^{3}$. Figure $6 \mathrm{~b}$ shows the electron transparency as a function of the ratio of the electric fields in the drift and the amplification volumes. The $\mathrm{V}_{\text {mesh }}$ was kept constant at $-315 \mathrm{~V}$. A scan in the 2 dimensional phase space of the $V_{\text {drift }} v s V_{\text {mesh }}$ (Figure 7) was performed for the determination of the optimal electric field settings in order to achieve the best energy resolution. Figure 7 shows that the optimal detector settings are around the values: $\mathrm{V}_{\text {drift }}=-355 \mathrm{~V}$ and $\mathrm{V}_{\text {mesh }}=-325 \mathrm{~V}$. One of the detectors presented excellent energy resolution $\Delta \mathrm{E}=11.5 \%$ at $\mathrm{FWHM}$ at $5.9 \mathrm{keV}$ (see Figure 8). The energy resolution depends on the Fano factor $(\mathrm{F}=0.20$ for $\operatorname{Ar}[6])$ and the Gain fluctuations factor (parameter $\mathrm{b}=0.30$ for microbulk [7, paragraph 4.4.2]) i.e. $\Delta E / E=2.35 \sqrt{w(F+b) / E}=11 \%$ $(\mathrm{w}=26.2 \mathrm{eV}$ and $\mathrm{E}=5900 \mathrm{eV})$. The measured value is close to the achievable limit of $11 \%$, with microbulk detectors at this energy. Other detectors with the same exactly characteristics presented a degraded energy resolution (up to $23 \%$ FWHM). This fact happened due to misalignments between the $\mathrm{x}$ and the $\mathrm{y}$ mesh strips which resulted in a bad charge collection. The problem was found by optical inspection with a microscope.
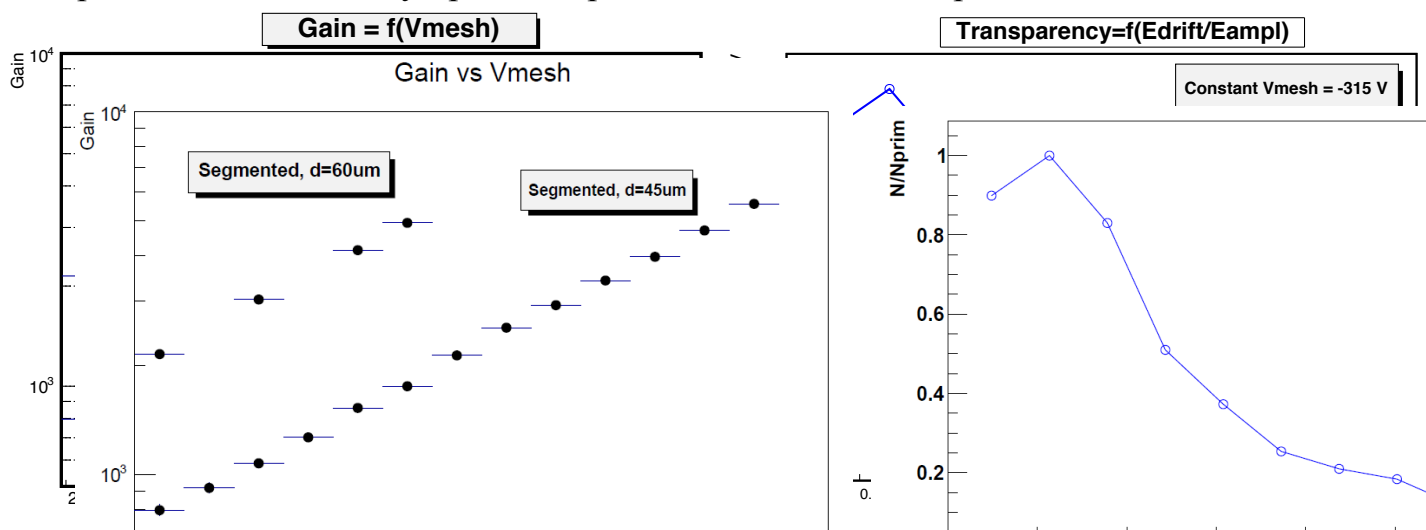

Figure 6 Left: The gain as a function of the $\mathrm{V}_{\text {mesh }}$ for two types of detectors. Right: The electron transparency curve as a function of the electric fields ratio $\mathrm{E}_{\text {driff }} / \mathrm{E}_{\text {amplification. }}$

$$
\text { Resolution=f(Vdrift,Vmesh) }
$$

\begin{tabular}{|l|l|}
\hline $\begin{array}{l}\text { Vmesh } \\
(\mathrm{V})\end{array}$ & $\begin{array}{l}\text { Vdrift (V) } \\
=\text { Vmesh }+\end{array}$ \\
\hline 300 & +10 \\
\hline 310 & +20 \\
\hline 320 & +30 \\
\hline 330 & +40 \\
\hline 340 & +50 \\
\hline
\end{tabular}

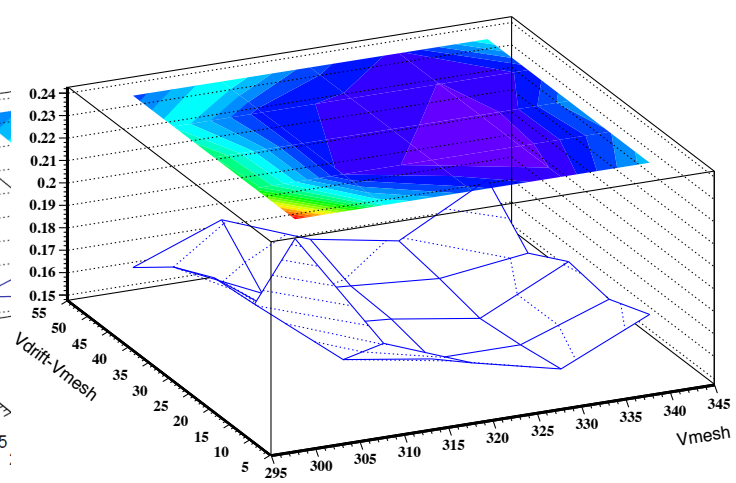

Figure 7 The energy resolution as a function of the $V_{\text {mesh }}$ and the $V_{\text {drift }}$ Optimal energy resolution values are located at the minimum (dark blue area). 


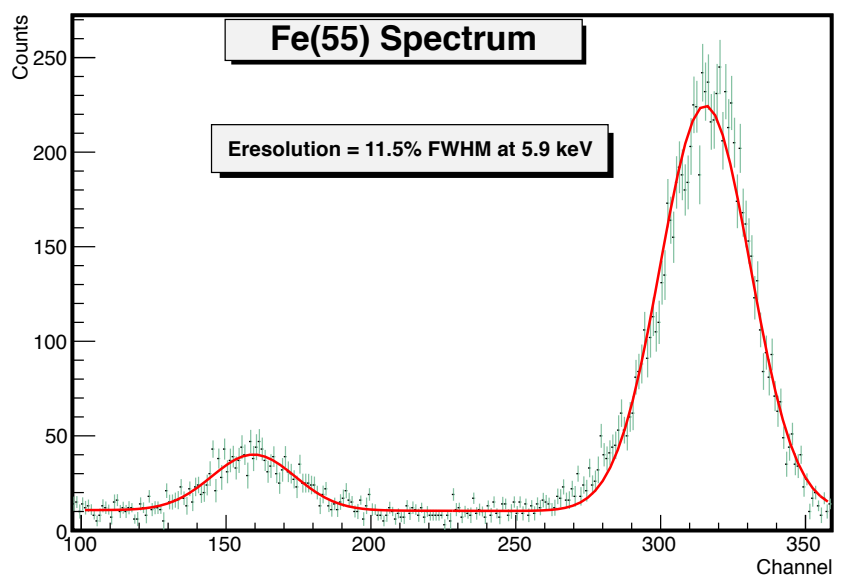

Figure 8 . The ${ }^{55} \mathrm{Fe}$ spectrum acquired with a segmented microbulk micromegas. The energy resolution is $11.5 \%$ at FWHM at $5.9 \mathrm{keV}$. The Argon escape peak at $3 \mathrm{keV}$ is clearly separated.

\section{3 $X$ and $Y$ strips readout with the Gassiplex FE card}

Micromegas detectors with one compact mesh, can provide the global mesh signal as a trigger for the readout. The segmented mesh microbulk requires the use of auto-triggering electronics. As previously mentioned we plan to use in the future the AGET electronics. As already described in paragraph 4.1 we can use the common y strips signal, in the "regular" HV setting, as a trigger to readout the $\mathrm{x}$-strips and vice versa for the y strips in "reversed" $\mathrm{HV}$. We used a VME based data acquisition [8], consisting of the V2718 CAEN VME controller, a VME gate generator, a V551 sequencer card, a CRAMS V550B card, NIM modules (Amplifier, Discriminator, HV power supply etc) and the Gassiplex FE card [9]. Figure 9 shows two events from the DAQ Event display, the y-Cluster multiplicity, the y-Cluster strip multiplicity and the spectrum from the ${ }^{55} \mathrm{Fe}$ source. The events are consistent with the photoelectron path in the gas (about $1 \mathrm{~mm}$ ) and typically they fire 1 or 2 strips. The plots for the x-strips are similar to the ones for the y-strips. We present in Figure 10 the $\mathrm{x}$-hit map, the x-cluster distribution and the ${ }^{55} \mathrm{Fe}$ energy spectrum as acquired by the strips analysis. We have performed a pedestal subtraction, cluster reconstruction and have finally calculated the Energy and the position of the cluster.
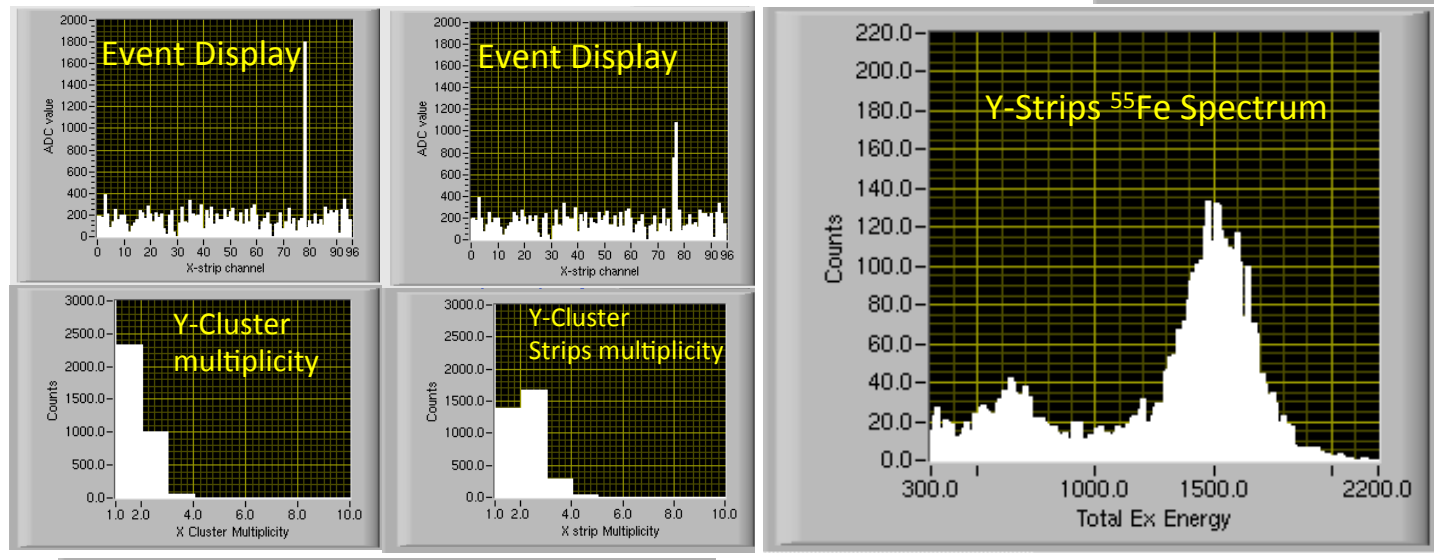

Figure 9. Event Display, y-cluster multiplicity, y-cluster strip multiplicity and the ${ }^{55} \mathrm{Fe}$ spectrum. 

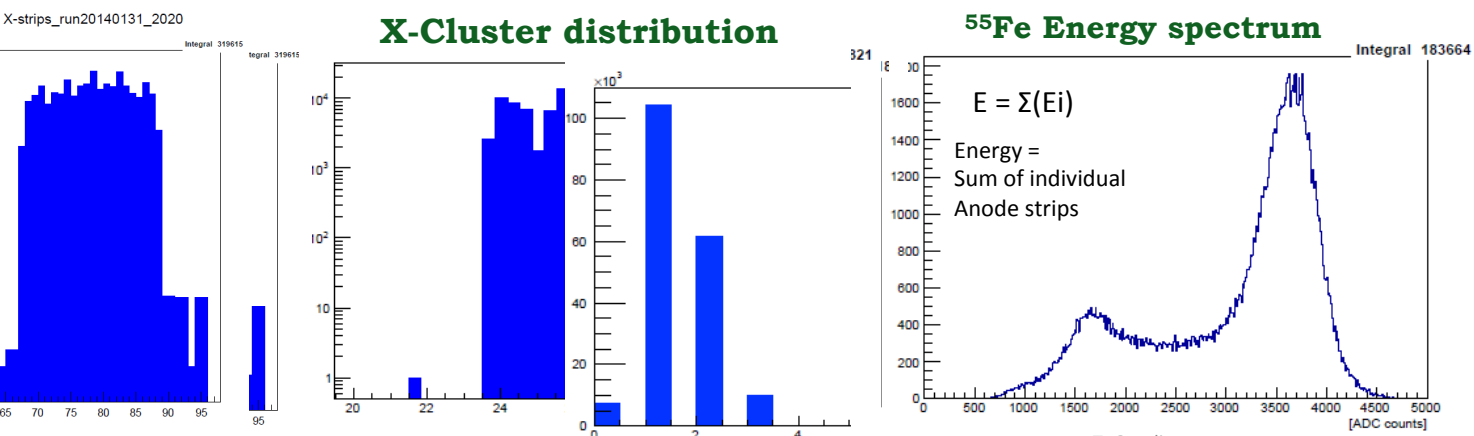

Figure 10. The $\mathrm{x}$-strips hit-map distribution, the $\mathrm{x}$-cluster distribution and the $55 \mathrm{Fe}$ energy spectrum from the $\mathrm{x}$-strip cluster information.

\section{Conclusion}

We have successfully built and operated for the first time segmented mesh micromegas based on the microbulk technology. We have demonstrated that their manufacturing is feasible and that the produced detectors possess excellent properties concerning energy resolution. All strips in $\mathrm{x}$ (anode) and $\mathrm{y}$ (mesh) direction are operational and provide the spatial information in both dimensions. There is good understanding of the cause of variations in their behavior and this will help the standardization in their manufacturing process. Axion and Dark matter searches are among the activities that can profit from this development thanks to the low material budget of the segmented microbulk and thus the expected improvement on the background reduction. Segmented microbulk micromegas is planned to be used in the nTOF experiment as neutron beam profiler offering a great neutron detector thanks again to its very low material.

\section{Acknowledgements}

We would like to thank the RD51 collaboration for partially supporting this project through the RD51 Common Fund. This research work was also supported, in part, by the program MIS-448332-ORASY (NSRF 2007 - 13 ACTION, KRIPIS) at INPP/NCSR Demokritos, Greece, the ERC T-REX Starting Grantref. ERC-2009-StG-240054 of the IDEAS program, 7th EU FP and the JIUZ-2013-CIE-02 project of the Universidad de Zaragoza, Spain. F.J. Iguaz acknowledges the support from the Juan de la Cierva programa.

\section{References}

[1] S. Andriamonje et al., Development and performance of Microbulk Micromegas detectors, JINST 5:P02001, 2010

[2] CAST Collaboration, Probing eV-scale axions with CAST, JCAP 0902:008, 2009

[3] S. Aune et al., Low background $x$-ray detection with Micromegas for axion search, JINST 9 (2014) P01001.

[4] http://www.comsol.com

[5] S. Anvar et al., AGET, the Get Front-End ASIC, for the readout of the TPC used in Nuclear Physics Experiments, NSS/MIC/RTSD IEEE, 2011.

[6] M. Kase, Fano factor in pure Argon, NIMA, Vol.227, Issue 2, 15 Nov. 1984, p311-317

[7] A. Th. Alquezar, PhD Thesis, Study of Time Projection Chambers with Micromegas for Rare Event Searches, http://zaguan.unizar.es/record/12540/files/TESIS-2013-098.pdf

[8] T. Geralis et al., The data acquisition system of the Micromegas detector for the CAST experiment, NSS 2003 IEEE, Oct. 2003, p3455 - 3459, Vol.5.

[9] J.-C Santiard et al., A low noise signal processor for readout of gaseous detectors, CERN-ECP/9417. 